\title{
Endoscopic surgery in the treatment of crista galli pneumatization evolving with localizated frontal headaches
}

\author{
Jan Alessandro Socher', Pedro Geisel Santos², Vinicius Cidral Correa ${ }^{3}$, Leandro Caetano de Barros e Silva ${ }^{3}$. \\ 1) Doctor of Otolaryngology Faculty of Medicine, University of Sao Paulo. Professor of Otorhinolaryngology in FURB - Regional University of Blumenau. \\ 2) Otolaryngologist. \\ 3) Student of Medicine in FURB - Regional University of Blumenau. \\ Institution: FURB - Regional University of Blumenau. \\ Blumenau / SC - Brazil \\ Mailing address: Jan Alessandro Socher - Alameda Duque de Caxias, 145 - sala 306 - Bairro Centro - Zip code: 89015-010 - Blumenau / SC - Brazil - E-mail: \\ jan_socher@yahoo.com.br \\ Article received on November $19^{\text {th }}, 2012$. Article accepted on March $19^{\text {th }}, 2013$.
}

\section{SUMMARY}

Introduction: The crista galli is part of the ethmoid bone and thus may suffer from the process of pneumatization. Pneumatization occurs in between $3 \%$ and $14 \%$ of patients, resulting from air cells in the frontal or ethmoid sinuses.

Aim: To describe 3 cases of crista galli pneumatization in which the patients developed infection and were treated surgically by endoscopic techniques.

Method: We present 3 case studies of patients complaining of severe frontal headaches. The patients underwent ENT evaluation, examination by video-endoscopy, and computed tomography, which identified crista galli pneumatization with mucosal thickening and the presence of fluid. Patients underwent treatment with antibiotics and corticosteroids; however, they showed no symptomatic improvement, displayed recurrence of symptoms, and maintained radiographic changes. Thus, patients then underwent drainage through the crista galli via an endoscopic procedure.

Discussion: During surgery, mucopurulence and/or mucosal thickening and edema were identified in the pneumatized crista galli. There were no complications during or after surgery. Postoperatively, headache was improved in patients after a minimum follow-up of 6 months.

Conclusion: Crista galli pneumatization can result in infection, simulating rhinosinusitis. When there is little response to drug therapy, endoscopic surgical treatment is required; the current cases demonstrate that this technique is safe and effective.

Keywords: Natural Orifice Endoscopic Surgery; Video-Assisted Surgery; Headache.

\section{INTRODUCTION}

The crista galli lies on the midline of the cribriform plate. The falx cerebri attaches anteriorly tothis bone formation a thin posterior border and slightly curve , and the anterior border is attached to the frontal bone completing the margin of the foramen cecum. The crista galli is embryologically derived from the ethmoid bone (1). Regarding pneumatization of the crista galli, 2 theories are valid: pneumatization can originate from the ethmoid sinus or the frontal sinus. The oldest theory in which the pneumatization result from the ethmoid sinus is based on the embryological origin of the crista galli, the ethmoid bone, and states that the displacement of ethmoidal air cells would lead to increased aeration of the crista galli ${ }^{2}$. In the theory explaining pneumatization by the frontal sinus, sinus extension is likely to cause increased aeration beyond the normal margin of the frontal bone (3). The incidence of crista galli pneumatization has been reported to be between $2.8 \%$ and $14.1 \%$, depending on the population studied $(2,4,5)$. The possibility of involvement of inflammatory and/or infectious processes in pneumatization of the crista galli is a noteworthy finding; however, such involvement is very rare since there are no reports of it in the literature.

\section{AIM}

The aim of this study was to describe 3 cases where crista galli pneumatization evolved into inflammation and infection and was treated surgically by endoscopic techniques.

\section{CASe Study \# I}

A 57-year-old female patient sought advice from the Specialized Service in Otolaryngology in June 2008, complaining of a frontal headache that had been present for the last year. The patient reported previous drug treatments for sinusitis including azithromycin for 5 days, amoxicillin for 10 days, and a combination of amoxicillin 


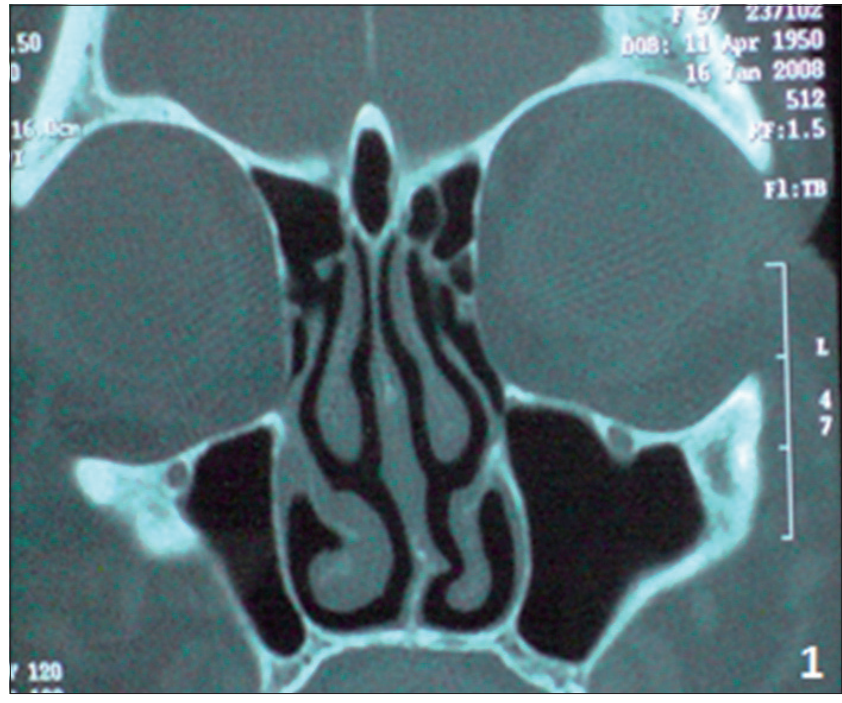

Figure 1. Preoperative coronal section CT scan showing crista galli pneumatization with mucosal thickening.

and clavulanate potassium for 10 days. However, she always experienced recurrence and progressive worsening of her symptoms. Examination with video-endoscopy showed hypertrophy of the lower and middle turbinates bilaterally associated with septal deviation in the left nasal cavity. A computed tomography (CT) scan of the paranasal sinuses and nasal cavity in the axial, coronal, and sagittal planes with a bone window of 2500-3500 rads was requested, which identified pneumatization of the crista galli with mild mucosal thickening in the interior (Figure 1). This finding indicated endoscopic surgery through the transseptal approach in the left nasal cavity for drainage and cleaning of the pneumatized crista galli (Figure 2).

\section{Case Study \#2}

A 35-year-old female patient sought advice from the Specialized Service in Otolaryngology in August 2010, complaining of a localized headache, cacosmia, and nasal obstruction with progressive worsening of her symptoms during the previous 6 months. Her symptoms had remained after drug treatment with amoxicillin and clavulanate potassium for 10 days. Video-endoscopy showed a deviated septum and inferior turbinate hypertrophy. A CT scan of the paranasal sinuses and nasal cavity in the axial, coronal, and sagittal planes with a bone window of 2500-3500 rads was requested, which identified pneumatization of the crista galli apophysis with signs of mucosal thickening and obliteration interiorly. We introduced antibiotic treatment (levofloxacin at a dose of $500 \mathrm{mg} /$ day) for 14 days, corticosteroids (prednisone at a dose of $40 \mathrm{mg} /$ day) for 7 days, and symptomatic analgesia. The patient's symptoms

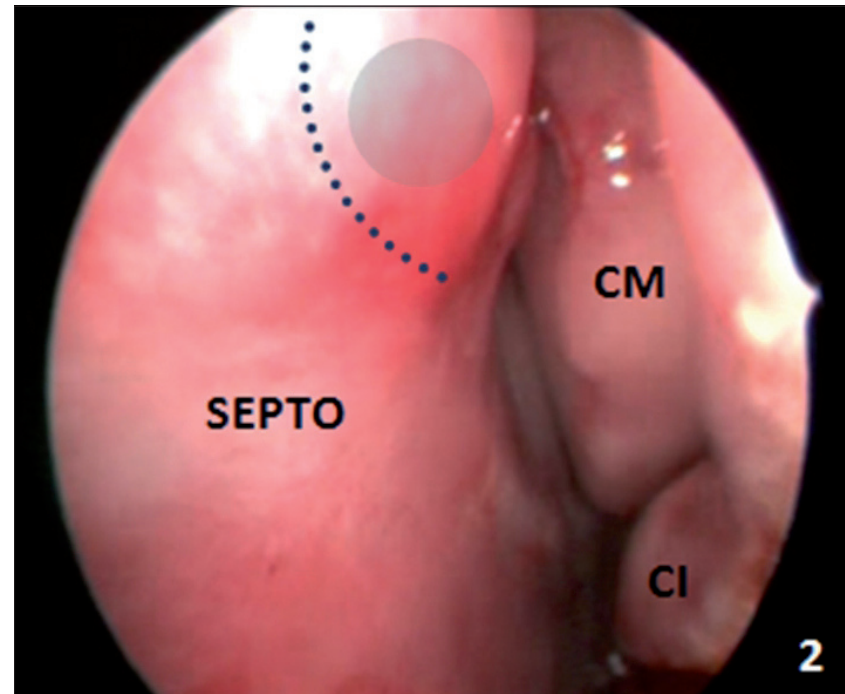

Figure 2. Video-endoscopic visualization of the left nasal cavity showing the septal incision site and opening of the bone wall of the pneumatized crista galli. SEPTO = nasal septum; $\mathrm{CM}=$ middle turbinate; $\mathrm{CI}=$ inferior turbinate.

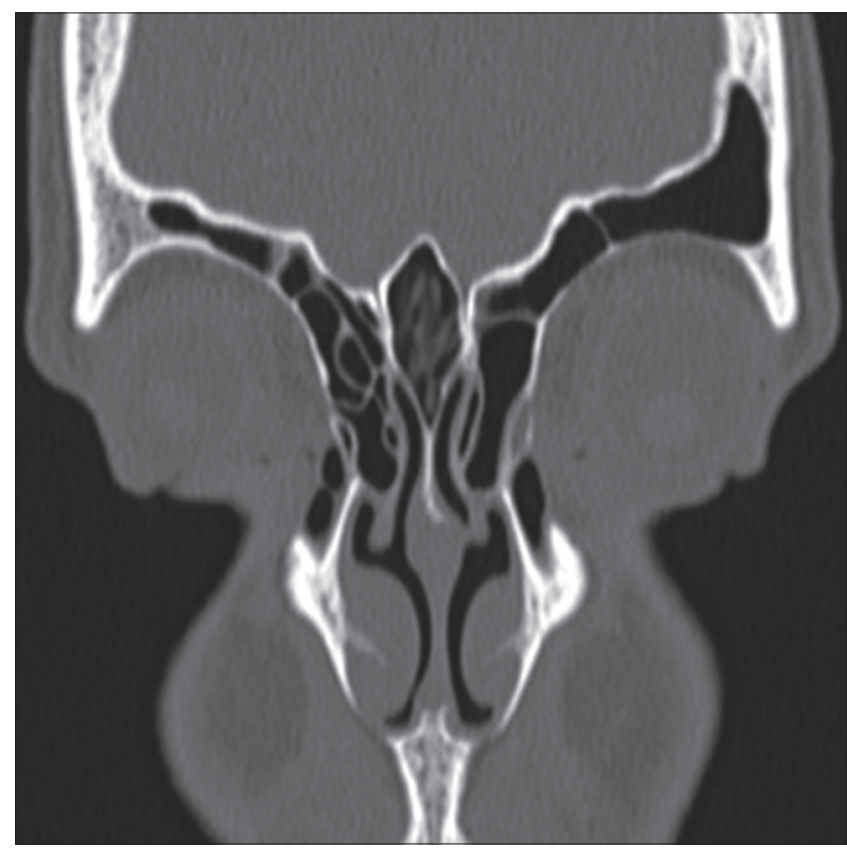

Figure 3. Coronal CT section showing mucosal thickening and obliteration of the pneumatized crista galli.

improved temporarily, but 5 days after treatment with antibiotics was stopped, recurrence of her symptoms occurred. A second CT scan of the paranasal sinuses and nasal cavity showed the maintenance of mucosal thickening and obliteration within the crista galli (Figure 3). Endoscopic surgery by a transseptal approach through the right nostril was indicated for drainage and cleaning of the pneumatized crista galli (Figures 4-7). 


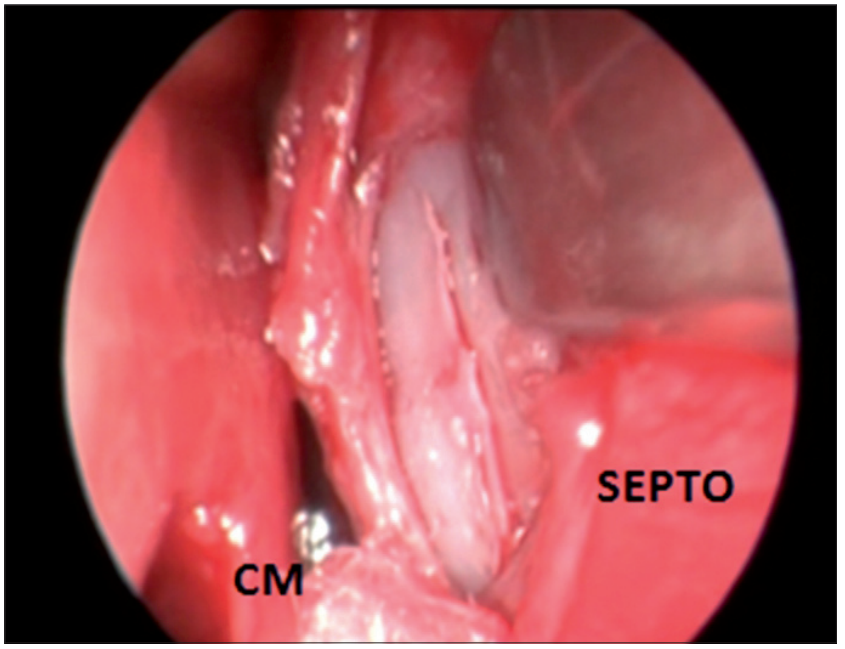

Figure 4. Intraoperative endoscopic visualization of the right nasal cavity showing septal incision and detachment. $\mathrm{SEPTO}=$ nasal septum; $\mathrm{CM}=$ middle turbinate .

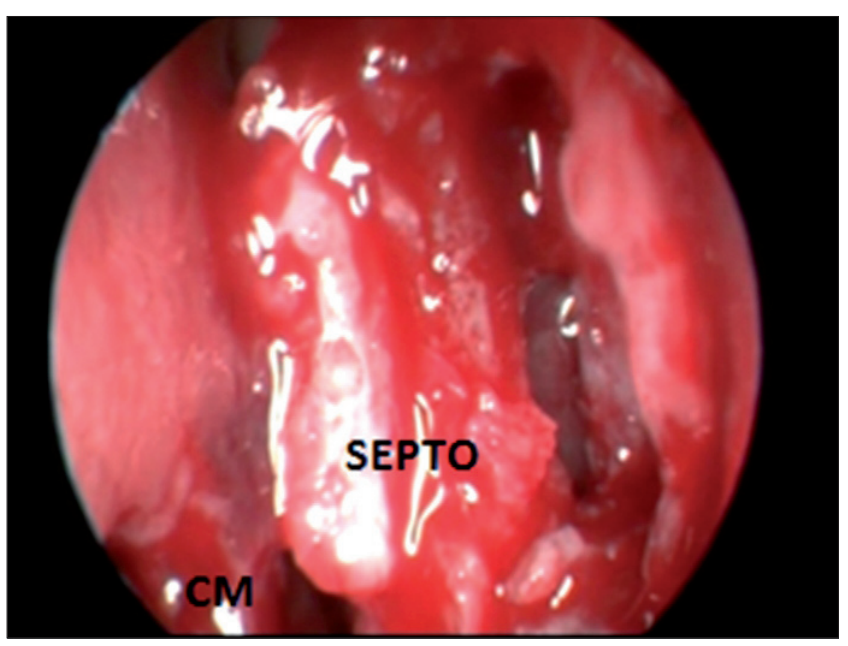

Figure 6. Intraoperative endoscopic visualization of the right nasal cavity showing transseptal opening of the bone wall of the pneumatized crista galli.

$\mathrm{SEPTO}=$ nasal septum; $\mathrm{CM}=$ middle turbinate .

\section{CASE Study \# 3}

A 31-year-old male patient sought advice from the Specialized Service in Otolaryngology in April 2012, with the complaint of chronic nasal obstruction that had been present since childhood and was associated with the symptoms of congestion, rhinorrhea, and facial pain localized in the frontal region for the last 3 months. He had shown no improvement after 2 previous episodes of treatment with antibiotics (levofloxin and amoxicillin lasting 10 days and 14 days, respectively). He also reported a history of adenoidectomy surgery prior to the

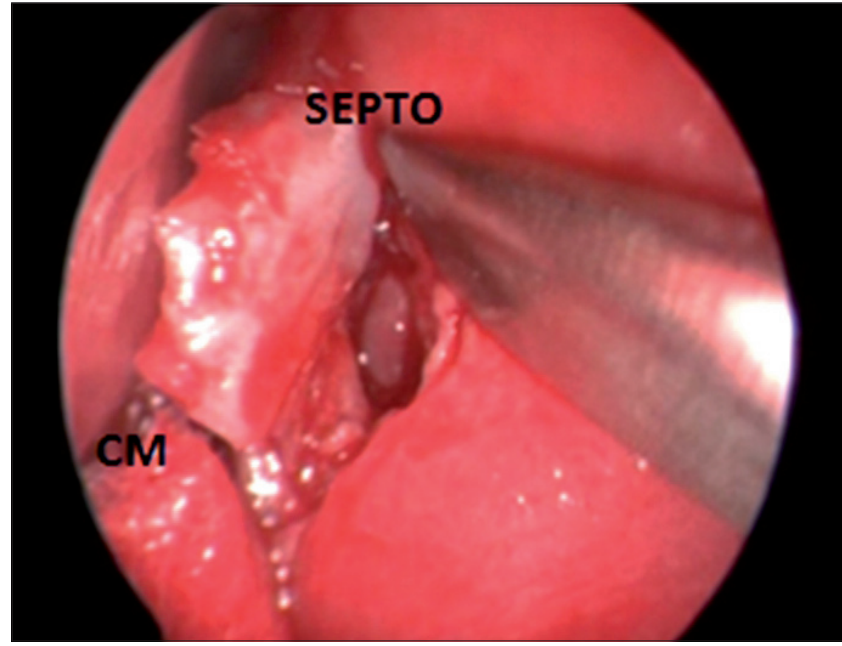

Figure 5. Intraoperative endoscopic visualization of the right nasal cavity demonstrating septal resection to allow approach to the bone wall of the pneumatized crista galli. $\mathrm{SEPTO}=$ nasal septum; $\mathrm{CM}=$ middle turbinate.

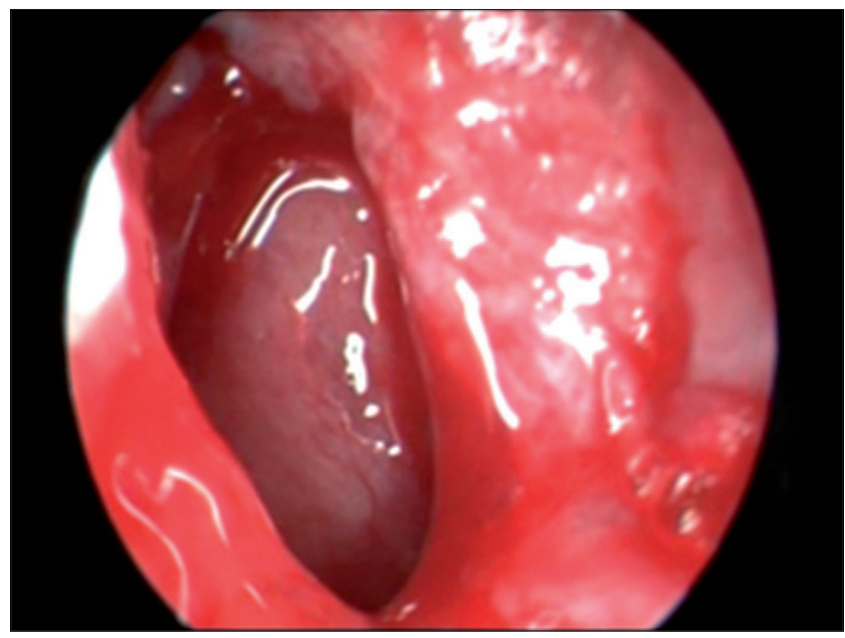

Figure 7. Intraoperative endoscopic visualization of the right nasal cavity demonstrating the detail of opening of the pneumatized crista galli, the lining of which shows mucosal thickening and edema.

age of 5. Video-endoscopic examination identified the presence of septal deviation in the left nasal cavity associated with mild inferior and right middle turbinate hypertrophy as well as hypertrophy of lymphoid tissue in the nasopharynx. A sinus and nasal cavity CT scan in the axial, coronal, and sagittal planes was requested, which identified septal deviation with bone spur formation, prominence of the soft parts of the cavum, and pneumatization of the crista galli with significant mucosal thickening and fluid present within (Figure 8). The patient underwent surgical resection of the lymphoid tissue present in the nasopharynx and transseptal endoscopic drainage of the pneumatized crista galli. 


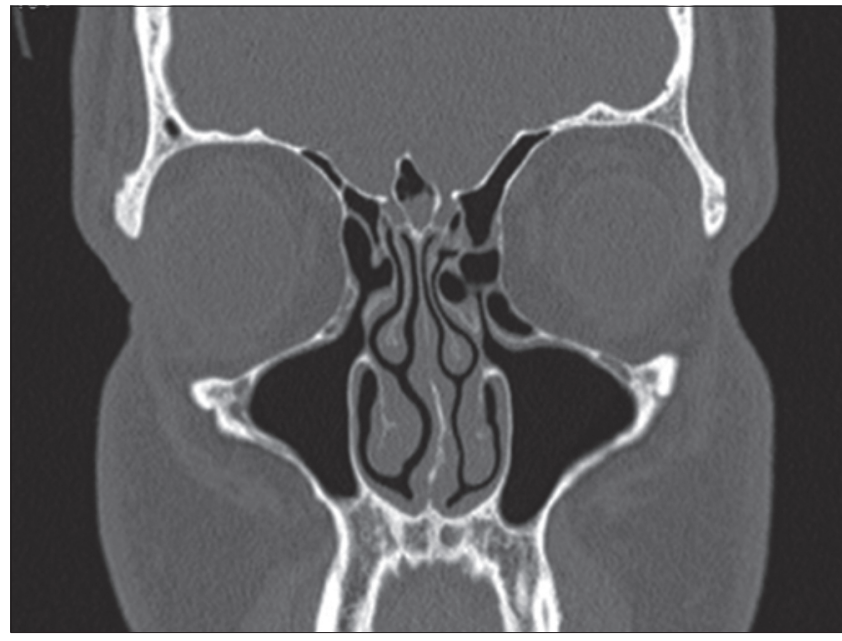

Figure 8. Coronal CT section showing mucosal thickening and fluid within the pneumatized crista galli.

\section{DISCUSSION}

Most studies on the crista galli in the literature are anatomical descriptions from surveys of series of CT scans or findings obtained during access to the anterior skull base using endoscopic surgery (1-5). There is a predominance in the study of pneumatization of the crista galli in females, with a ratio of 2 females: 1 male between the ages of 31 and 57 years. However, reports on pneumatization of the crista galli related to the complaint of headache were not found in the literature, and neither was evidence of the possibility of infection from such pneumatization.

In the present study, it was possible to access pneumatization of the crista galli and confirm the findings of CT scans showing signs of inflammation and/or infection in all 3 cases by using a transseptal endoscopic approach. In the first patient, mucosal thickening and edema was identified, while the second and third cases were associated with purulent drainage and identification of mucosal thickening and edema within the pneumatized crista galli. There were no transoperative or postoperative complications. However, it is important to note that good knowledge of the field of endoscopic technique and the anatomy of this region is essential, especially areas near the crista galli such as the cribriform plate and other structures of the anterior skull base. Patients were discharged on the same day as surgery, about 8 hours after operation. Antibiotics (oral levofloxacin at a dose of $500 \mathrm{mg}$ /day for 10 days in the first case and oral clindamycin at a dose of $900 \mathrm{mg} /$ day for 10 days in the other cases) plus corticosteroids (oral prednisone at a dose of $40 \mathrm{mg}$ /day for 7 days) were administered. Postoperative follow-ups were performed weekly during the first month and monthly for another 6 months thereafter. These follow-ups ensured maintenance of the patency of the crista galli through video-endoscopic examinations. After 6 months, the patients were advised to return annually or if there was a need.

The collected material was sent for bacterial and fungal culture, but the results were inconclusive, which can be explained by use of antibiotics prior to surgery. The use of different antibiotics prescribed based on clinical criteria for sinusitis was common in the history of all the patients prior to surgery. However, the use of antimicrobial agents was apparently unsuccessful in treating this condition, in view of the maintenance or recurrence of symptoms in all patients.

The findings of video-endoscopy examinations were nonspecific and did not contribute significantly to the diagnosis of these cases. The suspected inflammation and/ or infection within the pneumatized crista galli were only confirmed by CT scan.

In all cases, pathological results confirmed the presence of fragments of upper airway tract mucosa that were lined by pseudostratified ciliated epithelium resting on corium and contained a discrete amount of mononuclear inflammatory cells and eosinophils, which is characteristic of chronic inflammation of the respiratory mucosa contained inside the crista galli. Furthermore, pathological examination in the third patient confirmed the presence of lymphoid tissue in the nasopharynx that had no signs of malignancy and was compatible with pharyngeal tonsil.

The patients complained of transient postoperative nasal obstruction and hyposmia/anosmia, but showed improvement of symptoms and had no recurrence during a clinical monitoring period of 3 years in the first patient, 1 year and 6 months in the second patient, and 6 months in the third patient.

\section{CONCLUSION}

Pneumatization of the crista galli may be complicated by inflammatory and/or infectious processes simulating rhinosinusitis and shows little response to drug therapy, thus requiring endoscopic surgical treatment. In the current cases, such treatment was demonstrated to be safe and effective.

\section{REFERENCES}

1. Som PM, Park EE, Naidich TP, Lawson W. Crista Galli Pneumatization Is an Extension of the Adjacent Frontal Sinuses. AJNR Am J Neuroradiol. 2009;30:31-3. 
2. Hajiioannou J, Owens D, Whittet HB. Evaluation of anatomical variation of the crista galli using computed tomography. Clin Anat. 2010;23(4):370-3.

3. Miranda CMNR, Maranhão COM, Arraes FMNR, Padilha IG, Farias LPG, Jatobá MAS, Andrade ACM, Padilha BG. Variações anatômicas das cavidades paranasais à tomografia computadorizada multislice: o que procurar? Radiol Bras. 2011;44(4):256-62.
4. Dutra LD, Marchiori E. Tomografia computadorizada helicoidal dos seios paranasais na criança: avalização das sinusopatias inflamatórias. Radiol Bras. 2002;35(3):161-9.

5. Lee JM, Ransom E, Lee JYK, Palmer JN, Chiu AG. Endoscopic Anterior Skull Base Surgery: Intraoperative Considerations of the Crista Galli. Skull Base. 2011;21(2):836. 